\title{
1 Transfer learning via multi-scale convolutional neural layers for 2 human-virus protein-protein interaction prediction
}

3 Xiaodi Yang ${ }^{1}$, Shiping Yang ${ }^{2}$, Xianyi Lian ${ }^{1}$, Stefan Wuchty ${ }^{3,4,5^{*}}$ and Ziding Zhang ${ }^{1 *}$

$5 \quad{ }^{1}$ State Key Laboratory of Agrobiotechnology, College of Biological Sciences, China Agricultural

6 University, Beijing 100193, China

$7 \quad{ }^{2}$ State Key Laboratory of Plant Physiology and Biochemistry, College of Biological Sciences, China

8 Agricultural University, Beijing 100193, China

$9{ }^{3}$ Dept. of Computer Science, University of Miami, Miami, FL 33146, USA

$10 \quad{ }^{4}$ Dept. of Biology, University of Miami, Miami, FL 33146, USA

$11{ }^{5}$ Sylvester Comprehensive Cancer Center, University of Miami, Miami, FL 33136, USA

13 *Corresponding authors (Stefan Wuchty, Email: wuchtys@cs.miami.edu; Ziding Zhang, Email:

14 zidingzhang@cau.edu.cn;)

15

16 Other authors (Xiaodi Yang, Email: xiao di yang@163.com; Shiping Yang, Email:

17 shi ping yang@163.com; Xianyi Lian, Email: 1xy125760851@163.com)

18

19 


\section{Abstract}

21 To predict interactions between human and viral proteins, we combine evolutionary sequence

22 profile features with a Siamese convolutional neural network $(\mathrm{CNN})$ architecture and a multi-layer

23 perceptron (MLP). Our architecture outperforms various feature encodings-based machine learning

24 and state-of-the-art prediction methods. As our main contribution, we introduce two types of transfer

25 learning methods (i.e., 'frozen' type and 'fine-tuning' type) that reliably predict interactions in a

26 target human-virus domain based on training in a source human-virus domain, by retraining CNN

27 layers. Our transfer learning strategies can effectively apply prior knowledge transfer from large

28 source dataset/task to small target dataset/task to improve prediction performance. Finally, we utilize

29 the 'frozen' type of transfer learning to predict human-SARS-CoV-2 PPIs, indicating that our

30 predictions are topologically and functionally similar to experimentally known interactions. Source

31 code and datasets are available at https://github.com/XiaodiYangCAU/TransPPI/.

33 Keywords: human-virus relationship, protein-protein interaction, SARS-CoV-2, prediction, deep

34 learning, transfer learning 


\section{Introduction}

37 Viruses often employ a complex network of protein-protein interactions (PPIs) to coopt their own

38 cellular biological processes, strongly implying that the detection of virus-host PPIs is essential for

39 our understanding of the mechanisms that allow the virus to control cellular functions of the human

40 host. Considerable experimental efforts have been invested in the determination of binary

41 interactions between viral and human proteins, such as yeast two-hybrid (Y2H) assays and mass

42 spectroscopy (MS) technique (Calderwood et al., 2007; Dolan et al., 2013; Gordon et al., 2020; Shah

43 et al., 2019). However, maps of interactions between the human host and various viruses remain

44 incomplete, as a consequence of experimental cost, noise and a multitude of potential protein

45 interactions. Although tens of thousands of interactions have been determined through massive high-

46 and low-throughput experimental technologies, an immense need still exists for the development of

47 reliable computational methods to predict interactions between human and viral proteins.

48 The primary amino acid sequence remains the most easily to access and complete type of

49 protein information. As a consequence, many sequence-based feature extraction methods have been

50 developed, such as Local Descriptors (LD) (Davies et al., 2008; Yang et al., 2010), Conjoint Triads

51 (CT) (Shen et al., 2007; Sun et al., 2017) and Autocovariance (AC) (Guo et al., 2008; You et al.,

52 2013). Specifically, such features generally represent physicochemical properties or positional

53 information of amino acids that appear in the protein sequences. Based on these feature

54 characterization, several traditional machine learning algorithms (Cui et al., 2012; Dyer et al., 2011;

55 Eid et al., 2016; Emamjomeh et al., 2014; Lian et al., 2020) were previously applied to predict

56 interactions between human and viral proteins. While such machine learning methods effectively 
57 captured amino acid specific information to predict novel human-virus PPIs, large data sets

58 impaired their applicability through memory and runtime limits.

59 In the past decade, applications of deep learning methods have demonstrated improved

60 performance and potential in many fields (e.g., biomedicine, image, and speech recognition) (Karimi

61 et al., 2019; Pospisil et al., 2018; Sainath et al., 2015). In particular, convolutional neural networks

62 (CNNs) (Hashemifar et al., 2018) and recurrent neural networks (RNNs) (Zhang et al., 2016) are

63 comparatively well-established approaches, where CNNs automatically capture local features while

64 RNNs preserve contextualized/long-term ordering information. While deep learning methods

65 (Ahmed et al., 2018; Chen et al., 2019; Du et al., 2017; Hashemifar et al., 2018; Li et al., 2018; Sun

66 et al., 2017) have been designed to predict PPIs with excellent performance such models usually

67 focus on intraspecies interactions. In general, traditional machine learning/deep learning only

68 perform well, if training and test set are cut from the same statistical distribution in the feature space

69 (Shao et al., 2015). While the rigid application of a trained model on testing data sets with different

70 distributions usually perform poorly, transfer learning methods utilize prior knowledge from a 'source'

71 to train in a 'target' task domain (Chang et al., 2018; Shao et al., 2015) to improve performance.

72 Effective transfer learning can improve the generalization of models, reduce the amount of labeled

73 datasets, and save training time on the target dataset/task. In particular, transfer learning approaches

74 have been successfully applied to problems in many fields, such as medical imaging (Cheplygina et al.,

75 2019), biomedicine (Taroni et al., 2019), and visual categorization (Shao et al., 2015). With the

76 development of deep learning networks, a regular phenomenon appears in various training objectives

77 (Le et al., 2011; Lee et al., 2009) in that the first layers of deep neutral networks (DNNs) usually 
capture standard features of training data, providing a foundation for transfer learning. Specifically, a

79 deep neural network can be trained on a source task, establishing the parameters of the first layers.

80 Subsequently, parameters of late layers are trained on the target task, striking a balance between the

81 distributions of the different training domains. Depending on the size of the target dataset and number

82 of parameters of the DNN, first layers of the target DNN can either remain unchanged during training

83 on the new dataset or fine-tuned towards the new task, indicating a balance between specificity and

84 generality of derived prior knowledge (Taroni et al., 2019).

85 Here, we focus on the application of transfer deep learning approaches to the prediction of

86 interactions between proteins of viruses and the human host, an important issue amidst the world-wide

87 COVID-19 pandemic. In particular, we design a deep learning approach to predict interactions

88 between proteins of various viruses and the human host through representing interacting protein

89 sequences with a pre-acquired protein sequence profile module. In particular, we utilize Position

90 Specific Scoring Matrix (PSSM) features to encode the sequence characteristics with a Siamese-based

91 CNN that is fed to a multi-layer perceptron (MLP) (Figure 1) (see Materials and methods for details).

92 We propose two types of transfer learning methods through freezing/fine-tuning the parameters of the

93 CNN layers trained with a source and retrained with a target human-virus system to improve prediction

94 performance. Note that we trained the MLP layers with a target human-virus system through randomly

95 initializing parameters. Notably, we found that the transfer of prior knowledge learned from a

96 large-scale human-virus PPI dataset to predict interactions in smaller data sets such as Dengue virus,

97 Zika virus and severe acute respiratory syndrome coronavirus 2 (SARS-CoV-2) improved prediction

98 performance, provided better model generalization and reduced model training time. Finally, we 
99 employ the 'frozen' transfer learning models to predict human-SARS-CoV-2 PPIs based on pre-trained

100 models with all known human-viral protein interactions. Analysis of the obtained interaction network

101 indicates high functional similarity to experimentally observed host-virus interactions, suggesting that

102 our approach indeed provides meaningful predictions and can guide efforts to understand viral

103 infection mechanisms and host immune responses post SARS-CoV-2 infection.

\section{Results}

\section{The deep learning framework for human-virus PPI prediction}

107 Representing interactions between human and viral proteins through their sequences, we introduce an

108 end-to-end deep neural network framework, called a Siamese-based CNN that consists of a

109 pre-acquired protein sequence profile module, a Siamese CNN module and a prediction module

110 (Figure 1). First, we represent the amino acid composition of interacting proteins by protein sequence

111 profile (i.e., PSSM) features that capture evolutionary relationships. Such latent protein profile

112 representations of interacting protein pairs are fed to the Siamese CNN module to generate respective

113 high-dimensional sequence embeddings. In particular, our CNN module allows us to capture local

114 features of human and viral proteins such as protein linear binding motif patterns, which play

115 important roles in protein interactions. Finally, output embeddings of two proteins are combined to

116 form a sequence pair vector as the input to an MLP with appropriate loss function to predict the

117 presence/absence of an interaction between a viral and a human protein. Details about each module of

118 the deep neural network framework and the implementation details can be found in Materials and

119 methods. 


\section{Performance of the proposed deep learning method}

122 Based on our deep learning architecture we predicted interactions between proteins of various viruses

123 and the human host using 5-fold cross validation. In particular, we utilized 9,880 interactions in HIV,

124 5,966 in Herpes, 5,099 in Papilloma, 3,044 in Influenza, 1,300 in Hepatitis, 927 in Dengue and 709 in

125 Zika from different public databases (Altunkaya et al., 2017; Ammari et al., 2016; Calderone et al.,

126 2015; Durmuș Tekir et al., 2013; Guirimand et al., 2015), as well as 586 interactions of SARS-CoV-2

127 (Gordon et al., 2020; Li et al., 2020). As for negative sets, we employed 'Dissimilarity-Based Negative

128 Sampling' (Eid et al., 2016; Yang et al., 2020, 2021), and sampled non-interacting sets that were 10

129 times larger than the corresponding interaction data sets (see Materials and methods). While Table 1

130 indicates generally high prediction performance of our deep learning approach, we observed that small

131 sizes of training data sets such as Dengue, Zika and SARS-CoV-2 pointed to decreasing prediction

132 performance.

133 As random forest (RF) performs better than other machine learning methods when applied to

134 binary classification problems (Chen et al., 2019; Wu et al., 2009; Yang et al., 2020), we compare the

135 performance of our deep learning approaches (i.e., PSSM+CNN+MLP) to this representative

136 state-of-art classifier. Moreover, we consider three widely-used encoding methods (i.e., LD, CT and

137 AC) for feature representations as the input to the RF classifier using 5-fold cross validation. By

138 comparing AUPRC (area under precision-recall curve) values, we observed that our deep learning

139 method generally outperforms other encoding schemes-based RF classifiers especially when applied

140 to comparatively large datasets (Table 2). 
142 proteins, we compared the performance of our deep learning architecture based on PSSM to a different

143 word embedding technique. Specifically, the word2vec encoding method (Chen et al., 2019; Le and

144 Mikolov, 2014) considers each amino acid as a word, and learns a word-embedding of sequences

145 based on a large corpus, where each amino acid is encoded by a 5-dimensional vector. As 20 amino

146 acids are clustered into 7 groups based on their dipoles and volumes of the side chains (Shen et al.,

147 2007; Sun et al., 2017), that were used for the construction of conjoint triads (CT) we one-hot

148 encoded each amino acid of the corresponding sequence. As a result, word2vec+CT one-hot is the

149 concatenation of pre-trained amino acid embeddings where each protein is represented by a $n \times 12$

150 dimensional array. As noted previously, we considered a fixed sequence length of $n=2,000$ and

151 zero-padded smaller sequences. Training our CNN+MLP approach with word2vec+CT one hot

152 encodings of the corresponding protein sequences, we observed that the representation of sequences

153 through PSSM in our approach provided better prediction performance especially in relatively small

154 datasets such as Dengue, Zika and SARS-CoV-2 (Table 3).

156 Comparison with other existing human-virus PPI prediction methods

157 To further assess the performance of our proposed method, we compared our method with three

158 existing human-virus PPI prediction approaches. Recently, we proposed a sequence embedding-based

159 RF method to predict human-virus PPIs with promising performance (Yang et al., 2020). In particular,

160 we applied an unsupervised sequence embedding technique (i.e., doc $2 \mathrm{vec}$ ) to represent interacting

161 protein sequences as low-dimensional vectors with rich features that are subjected to RF to predict the 
162 presence/absence of an interaction. In Alguwaizani et al.'s work (Alguwaizani et al., 2018), the

163 authors utilized a Support Vector Machine (SVM) model to predict human-virus PPIs based on

164 feature-encoding of protein sequences through patterns of repeats and local amino acid combinations.

165 As for the DeNovo method (Eid et al., 2016), the authors introduced a domain/linear motif-based

166 SVM approach to predict human-virus PPIs. To make a fair comparison, we first constructed the

167 PSSM of the protein sequences of DeNovo's PPI dataset and used their training set to train our

168 Siamese-based CNN model. Finally, we assessed the performance of our reconstructed deep learning

169 model on the test set provided in (Eid et al., 2016) including 425 positive and 425 negative samples.

170 Furthermore, we evaluated the performance of our previous RF based prediction method and

171 Alguwaizani et al.'s SVM approach with these data sets as well. Notably, Table 4 suggests that our

172 deep learning and our previously published RF-based method clearly outperformed other approaches.

\section{Cross-viral tests and transfer learning}

175 To explore potential factors that affect prediction performance in a cross-viral setting, we trained our

176 deep learning model on one human-virus PPI data set and predicted protein interactions in a different

177 human-virus system. Utilizing 5-fold cross validation, we expectedly observed that the performance of

178 such naïve cross-viral tests dropped considerably compared to training and testing in the same

179 human-virus system (Figure 2a). To allow reliable cross-viral predictions of PPIs, we introduce two

180 transfer learning methods (i.e., 'frozen' and 'fine-tuning') where we first train the parameters of CNN

181 layers on a source human-virus PPI dataset. Subsequently, we transferred all parameters of CNN layers

182 to initialize a new model ('frozen' or 'fine tuning') with randomly initialized MLP layers to train on a 
183 given target human-virus PPI domain. To comprehensively test our transfer learning approaches, we

184 considered each combination of human-viral PPI sets as source and target domains. Figure 2b

185 indicates that a relatively rigid transfer learning methodology by keeping the parameters of the CNN

186 module untouched (i.e., 'frozen') and training the MLP layers strongly outperformed the naïve

187 baseline performance as shown in Figure 2a. In turn, fine-tuning parameters in the CNN module and

188 training the MLP layers as well with a given target human-viral domain allowed for another increase in

189 performance (Figure 2c). As for individual pairs of human-viral domains, we also observed that

190 independently from the training domain the 'frozen' transfer methodology worked better compared to

191 the 'fine-tuning' approach when the target domain data set was extremely small (i.e.,

192 human-SARS-CoV-2). In turn, performance of the 'frozen' transfer learning approach dropped

193 compared to 'fine-tuning' when the target human-viral domain datasets of PPIs were larger such as

194 human-Hepatitis, human-Dengue and human-Zika.

Prediction and analysis of human-SARS-CoV-2 PPIs based on transfer learning models

197 To predict a genome-wide map of potential interactions between proteins of the human host and

198 SARS-CoV-2, we first trained parameters of the CNN layers of our deep learning model utilizing all

199 human-viral protein interactions. Subsequently, we used our two transfer learning approaches to train

200 our set of interactions between proteins of human and SARS-CoV-2. Applying 5-fold cross

201 validations, we observed that the AUPRC of 0.483 with the 'frozen' transfer learning approach

202 outperformed the corresponding value of 0.435 when we used the 'fine-tuning' method. In addition,

203 training on all source human-virus PPI datasets showed best performance compared to separately 
204 training with virus-specific source PPI datasets (data not shown). Therefore, we employed the five

205 'frozen' models of the 5-fold cross-validation based on human-all virus source dataset to predict

206 human-SARS-CoV-2 PPIs and averaged the scores of the five models as the prediction result. At a

207 false positive rate control of 0.01 , we identified 946 high-confidence protein interactions between 21

208 SARS-CoV-2 proteins and 551 human proteins (Supplementary Table S1). As for topological

209 network analysis, we found a power-law distribution when we counted the number of human proteins

210 that were targeted by a certain number of viral proteins (Figure 3a). Such an observation suggests

211 that the majority of human proteins are targeted by one viral protein, while a minority of human host

212 proteins interacts with many viral proteins, a result that is in line with previous observations (Wuchty

213 et al., 2010). As targeted human proteins usually play important topological roles (i.e., hubs) in

214 human PPI networks, we performed an enrichment analysis of our identified viral targets by using a

215 human specific PPI network. In particular, we collected 365,284 human PPIs from the HIPPIE

216 database (Alanis-Lobato et al., 2017) and observed that targeted human proteins are enriched in bins

217 of increasing degree (Figure 3b), a result that is consistent with previous findings as well (Dyer et al.,

218 2008; Wuchty et al., 2010). Considering 2,916 human protein complexes from the CORUM database

219 (Giurgiu et al., 2019) we found that viral targets are enriched in sets of proteins that participate in an

220 increasing number of protein complexes (Figure 3c) (Wuchty et al., 2010). To illustrate viral

221 similarities, we compared the experimentally known human-SARS-CoV-2 interactome and our

222 predicted interactome with their counterparts of seven other viruses (Figure 3d). While our

223 predictions show similar overlaps of viral targets with the experimentally obtained interactomes, we

224 further found that Dengue virus and Influenza virus had the most similar interacting partners in both 
225 predictions and experimentally known interactions ( $P$-value $\leq 0.05$, hypergeometric test). Notably,

226 the association with Influenza virus is of particular interest as this virus also induces respiratory

227 disease (i.e., pneumonia).

229 General functional roles of SARS-CoV-2 targets

230 On a quantitative functional level, we calculated overlaps between both our predicted and known

231 viral targets and several functional gene sets [i.e., essential gene set, transcription factor set, kinases

232 set and posttranslational modification (PTM) set] to characterize the functions of viral targets

233 (Figure 4). As a result, we found that both predicted and known viral targets were significantly

234 enriched with essential genes (Figure 4a), while they rarely were transcription factors and kinases

235 (Figure 4b, c). In turn, we observed that ubiquitinated, methylated and acetylated proteins

236 increasingly appeared in sets of viral targets while phosphorylated target appeared not enriched

237 (Figure 4d-g). In particular, several studies have revealed SARS-CoV-2 targets human proteins that

238 receive PTMs relate to pathways that modulate host antiviral immune responses (Pruimboom, 2020;

239 Shin et al., 2020). In turn, phosphorylation appears ubiquitous and not specific for viral targets.

241 Comparative analysis of known and our predicted human-SARS-CoV-2 PPIs

242 Comparing our predicted and experimentally obtained sets of interactions between proteins of

243 SARS-CoV-2 and the human host, we found considerable overlaps. In particular, 298 out of 946

244 predicted PPI were identified through previous experimental efforts that amount to 52.5\% of known

245 interactions in SARS-CoV-2, while 648 were specifically identified through our deep learning 
246 approach (Figure 5a, Supplementary Table S1), indicating the reliability and specificity of our

247 model for the identification of novel interactions. To further excavate functional similarities and

248 differences between known and predicted targets, we performed functional and pathway enrichment

249 for experimentally known and predicted viral targets, respectively. Considering hypergeometric tests

250 (Bonferroni corrected $P$-value $\leq 0.01$ ), we observed a relatively large number of shared GO

251 enrichment terms/KEGG enrichment pathways of experimentally confirmed targets and predicted

252 targets, indicating functionally similar between them, which further demonstrates the reliability and

253 quality of our predictions. (Figure 5b, Supplementary Table S2, S3). In more detail, enriched GO

254 BP terms in human host proteins that were found in the experimental PPIs and predictions are

255 displayed in Figure 5c. Indicating high confidence of our predicted targets to discover functions

256 SARS-CoV-2 meddles in, we found that functional enrichments of experimental and predicted viral

257 targets both point to the involvement of viral targets in protein transport, protein import and mRNA

258 export from the nucleus. Notably, our predictions augment such functions, indicating that the virus

259 may also interfere with nuclear pore organization and assembly as well as protein export from the

260 nucleus.

\section{Modular analysis of human-SARS-CoV-2 PPI network}

263 To further explore potential functional modules that can reveal SARS-CoV-2 biology, we combined

264 our predicted 946 human-SARS-CoV-2 PPIs with known human-specific PPIs as of the HIPPIE

265 database (Alanis-Lobato et al., 2017) (Figure 6a). Specifically, we identified 9 topological modules

266 based on connectivity among human proteins (Figure 6a, b), utilizing the MCODE algorithm (Bader 
267 and Hogue, 2003). Investigating the enrichment of GO BP terms and KEGG pathways through

268 hypergeometric tests (Bonferroni adjusted $P$-value $\leq 0.05$ ), we observed that these modules largely

269 revolve around ribosome biogenesis, retrograde protein transport, elastic fiber assembly,

270 mitochondrial translation, protein processing in endoplasmic reticulum, stress granule regulation,

271 protein folding in endoplasmic reticulum, centrosome and gene splicing (Supplementary Table S4).

272 Considering a module that was enriched with centrosome functions through interactions with

273 nsp13 and cell cycle functions through interactions with orf6, we also found that this module harbors

274 human genes that allow SARS-CoV-2 to interact with innate immune pathways which is consistent

275 with previous findings (Gordon et al., 2020). As shown in the module, the interferon (IFN) pathway

276 is targeted though TBK1 by nsp8, nsp13 and orf6, a serine/threonine-protein kinase that plays an

277 important role in the induction of the antiviral IFN responses to foreign agents such as viruses. A

278 number of viral proteins binds to TBK1 and regulates its kinase activity to reduce TBK1-mediated

279 secretion of IFN and induction of an antiviral state, such as Borna disease virus (BDV) P protein

280 (Unterstab et al., 2005), Human herpesvirus 1 (HSV-1) ICTP34.5 protein (Manivanh et al., 2017) and

281 Ebola virus (EBOV) VP35 protein (Prins et al., 2009). BDV P protein itself is phosphorylated by

282 TBK1, suggesting that $\mathrm{P}$ functions as a viral decoy substrate that prevents activation of cellular target

283 proteins of TBK1. Furthermore, residues from 87 to 106 in HSV-1 ICTP34.5 protein interact with

284 TBK1 to modulate type I IFN signaling (Manivanh et al., 2017; Verpooten et al., 2009). Considering

285 the multiple sequence alignment of these viral proteins and nsp13 of SARS-CoV and SARS-CoV-2

286 we found a conserved binding motif (Figure 6c), corroborating our assumption that SARS-CoV-2

287 nsp13 protein may also interfere with the regulation processes of IFN that support antiviral innate 
288 immune response.

289 In a different module (Figure 6d), various virus proteins of SARS-CoV-2 target stress granule

290 regulation (N, nsp3, nsp12, nsp15), RNA processing (N) and protein export functions (M). Notably,

291 G3BP1 and G3BP2 are stress granule proteins with anti-viral activity. The nsp3 protein of several

292 other viral pathogens interacts with G3BP1 via C-terminus including Sindbis virus (SINV), Semliki

293 forest virus (SFV) and Chikungunya virus (CHIKV). Such interactions inhibit the formation of host

294 stress granules on viral mRNAs while the nsp3-G3BP1 complexes bind viral RNAs and probably

295 orchestrate the assembly of viral replication complexes. In particular, coronavirus commonly

296 manipulates the stress granules and related RNA biology processes while stress granule

297 formation/assembly is considered a primary antiviral response (Nakagawa et al., 2018;

298 Quispe-Tintaya, 2019). To corroborate such findings, we found a conserved amino-acid motif

299 ('FGXF') in the nsp3 proteins of these viruses. In particular, mutations in the 'FGDF' motif of SFV

300 nsp3 protein indicated that residues F, G, F are essential for G3BP-binding (Panas et al., 2015).

301 Notably, we found that the SARS-CoV-2 nsp12 protein and the SARS-CoV nsp3 protein both

302 contained the complete 'FGXF' motif, indicating the reliability of our prediction and the detailed

303 interaction pattern.

\section{Discussion and conclusions}

306 We designed a Siamese-based multi-scale CNN architecture by using PSSM to represent the sequences

307 of interacting proteins, allowing us to predict interactions between human and viral proteins with an

308 MLP approach. In comparison, we observed that our model outperformed previous state-of-the-art 
309 human-virus PPI prediction methods. Furthermore, we confirmed that the performance of the

310 combination of our deep learning framework and the representation of the protein features as PSSM

311 was mostly superior to combinations of other machine learning and pre-trained feature embeddings.

312 While we found that our model that was trained on a given source human-viral interaction data set

313 performed dismally in predicting protein interactions of proteins in a target human-virus domain in a

314 naïve way, we introduced two transfer learning methods (i.e., 'frozen' type and 'fine-tuning' type).

315 Such methods allowed us to train on a source human-virus domain and retrain the layers of CNN with

316 data of a target domain. Notably, our methods increased the cross-viral prediction performance

317 dramatically, compared to the naïve baseline model. In particular, for small target datasets, fine-tuning

318 pre-trained parameters that were obtained from larger source sets increased prediction performance.

319 Specifically, keeping the CNN layers from large source datasets untouched improved the

320 generalization ability of the new model (i.e., 'frozen' model), while retraining layers of CNN (i.e.,

321 'fine-tuning' model) allowed the specificity of predictions in target domain datasets.

322 Finally, we employed our 'frozen' transfer learning to predict human-SARS-CoV-2 PPIs and

323 performed in-depth network analysis based on the identified interactions. Our transfer learning model

324 resembled closely the functions and characteristics of experimentally obtained interactions and

325 indicated novel functions that the virus potentially targets. Furthermore, we identified several

326 high-confidence interactions involved in various vital immune pathways and anti-viral processes.

327 Taken together, our transfer learning method can be effectively applied to predict human-virus PPIs

328 in a cross-viral setting and the study of viral infection mechanism. 


\section{Materials and methods}

\section{Deep learning network architecture}

332 Pre-acquired protein sequence profile module. For each protein sequence with variable lengths, we

333 generated a sequence profile, called position specific scoring matrix (PSSM). In particular, we

334 performed PSI-BLAST searches with default parameters applying a threshold of E-value $<0.001$ in

335 the UniRef50 protein sequence database (Suzek et al., 2015) as PSI-BLAST allows us to discover

336 protein sequences that are evolutionarily linked to the search sequence (Hamp and Rost, 2015;

337 Hashemifar et al., 2018). Sequence profiles thus obtained for each search sequence were processed by

338 truncating profiles of long sequences to a fixed length $n$ and zero-padding short sequences, a method

339 widely used for data pre-processing and effective training (Matching, 2018; Min et al., 2017). As a

340 result, we obtained a $n \times 20$ dimensional array $S$ for each protein,

$$
S=\left[\begin{array}{ccccc}
s_{1,1} & \cdots & s_{1, j} & \cdots & s_{1,20} \\
\vdots & \cdots & \vdots & \cdots & \vdots \\
s_{i, 1} & \cdots & s_{i, j} & \cdots & s_{i, 20} \\
\vdots & \cdots & \vdots & \cdots & \vdots \\
s_{n, 1} & \cdots & s_{n, j} & \cdots & s_{n, 20}
\end{array}\right]
$$

capturing the probability $s_{i, j}$ that the residue in the $i^{\text {th }}$ position of the sequence is the $j^{\text {th }}$ out of the

343 alphabet of 20 amino acids.

$344 \quad$ Siamese CNN module. To capture complex relationship between two proteins we employ a

345 Siamese CNN architecture with two identical CNN sub-networks that share the same parameters for a

346 given pair of protein profiles $S, S^{\prime}$. Each sub-network produces a sequence embedding of a single

347 protein profile that are then concatenated. While each single CNN module consists of a convolutional

348 and pooling layer, we leveraged four connected convolutional modules to capture the patterns in an 
349 input sequence profile.

350 Specifically, we use $X$, a $n \times s$ array of length $n$ with $s$ features in each position. The 351 convolution layer applies a sliding window of length $w$ (the size of filters/kernels) to convert $X$ into 352 a $(n-w+1) \times f n$ array $C$ where $f n$ represents the number of filters/kernels. $C_{i, k}$ denotes the 353 score of filter/kernel $k, 1 \leq k \leq f n$, that corresponds to position $i$ of array $X$. Moreover, the 354 convolutional layer applies a parameter-sharing kernel $M$, a $f n \times m \times s$ array where $M_{k, j, l}$ is the 355 coefficient of pattern $k$ at position $j$ and feature $l$. The calculation of $C$ is defined as

$$
\begin{gathered}
C=\operatorname{Conv}_{M}(S) \\
C_{i, k}=\sum_{j=1}^{m} \sum_{l=1}^{s} M_{k, j, l} X_{i+j, l}
\end{gathered}
$$

356 Furthermore, the pooling layer is utilized to reduce the dimension of $C$ to a $(n-p+1) \times f n$

357 array $P$ where $p$ is the size of pooling window. Array $P=\operatorname{Pool}(C)$ is calculated as the maximum of

358 all positions $i \leq j \leq i+p$ over each feature $k$ where $1 \leq i \leq(n-m+1)-p$,

$$
P_{i, k}=\operatorname{Max}\left(C_{i, k}, \ldots, C_{i+l, k}\right)
$$

Prediction module. The prediction module concatenates a pair of protein sequence embedding

vectors into a sequence pair vector as the input of fully connected layers in an MLP and computes the

361 probability that two proteins interact. The MLP contains three dense layers with leaky ReLU where

362 cross-entropy loss is optimized for the binary classification objective defined as

$$
\text { Loss }=-\frac{1}{|K|} \sum_{p \in K} \sum_{i=1}^{m} y_{i}^{p} \log s_{i}^{p}
$$

where $y_{i}$ is numerical class label of the protein pair $p$. The output of the MLP for the protein pair

$364 p$ is a probability vector $\hat{s}^{p}$, whose dimensionality is the number of classes $m . s$ is normalized by a 
365 softmax function, where the normalized probability value for the $i^{t h}$ class is defined as $s_{i}^{p}=$ $366 \exp \left(\hat{s}_{i}^{p}\right) / \sum_{j} \exp \left(\hat{S}_{j}^{p}\right)$

367 Implementation details. As for pre-acquired sequence profile construction, we consider a fixed

368 sequence length of 2,000. As for the construction of our learning approach, we employ four

369 convolutional modules, with input sizes $20,64,128$ and 256 . The convolution kernel size is set to 3

370 while the size of pooling window is set to 2 with 3 max-pooling layers and a global max-pooling layer.

371 To optimize the cross-entropy loss function we used AMSGrad (Reddi et al., 2018) and set the learning

372 rate to 0.0001 . The batch size was set to 64 , while the number of epochs was 100 . The fully connected

373 layers contained three dense layers with input size 1,024, 512, 256 and output a two-dimensional

374 vector with the last softmax layer. The whole procedure was implemented with keras (https://keras.io/)

375 with GPU configuration.

\section{Data set construction}

378 We collected experimentally verified human-virus PPI data capturing 31,381 interactions in all viruses.

379 Specifically, we accounted for 9,880 interactions in HIV, 5,966 in Herpes, 5,099 in Papilloma, 3,044 in

380 Influenza, 1,300 in Hepatitis, 927 in Dengue and 709 in Zika from five public databases, including

381 HPIDB (Ammari et al., 2016), VirHostNet (Guirimand et al., 2015), VirusMentha (Calderone et al.,

382 2015), PHISTO (Durmuş Tekir et al., 2013) and PDB (Altunkaya et al., 2017). As for interactions of

383 proteins of SARS-CoV-2, we used two recently published interaction sets (Gordon et al., 2020; Li et al.,

384 2020) that captured 291 and 295 PPIs, respectively. To obtain high-quality PPIs, we removed

385 interactions from large-scale MS experiments that were detected only once, non-physical interactions 
386 parameter selection and optimization is shown in Supplementary Table S5.

and interactions between proteins without available PSSM features. a negative sample.

\section{Two types of transfer learning methods}

Sampling negative interactions, we employed a 'Dissimilarity-Based Negative Sampling' method

(Eid et al., 2016) as outlined in our previous work (Yang et al., 2021, 2020) and set the ratio of pos./neg.

samples to 1:10. The key strategy of the 'negative sampling' is if the sequences of viral protein A and B

are similar, and A interacts with human protein C (i.e., A-C is a positive sample), B-C is not selected as

To further improve the performance of our deep neural network especially when dealing with smaller datasets, we propose two transfer learning methods that keep the parameters of the CNN layers constant (i.e., 'frozen') or allow their fine-tuning in the early layers (i.e., 'fine-tuning') and applied them to eight human-virus PPI sets. In more detail, we used the proposed DNN architecture to train the models based on a given source set of human-virus interactions to obtain pre-trained parameters in the CNN layers that learn the representation of the protein sequences. In subsequent transfer learning steps, we keep the parameters of these CNN layers constant (i.e., frozen) and only train parameters of the fully connected layers of the MLP to predict interactions in a target human-viral interaction set. As an alternative, our 'fine-tuning' approach trains parameters of the fully connected layers of the MLP and retrains the parameters of $\mathrm{CNN}$ layers that we obtained from the initial training step and change such parameters by learning the interactions in a target set of human-virus interactions. The detailed 


\section{Alternative machine learning and feature encoding methods}

409 Random Forest. RF (Hamp and Rost, 2015; Wu et al., 2009) is an ensemble learning method where

410 each decision tree is constructed using a different bootstrap sample of the data ('bagging'). In addition,

411 RF changes how decision trees are constructed by splitting each node, using the best among a subset of

412 predictors randomly chosen at that node ('boosting'). Compared to many other classifiers this strategy

413 turns out to be robust against over-fitting, capturing aggregate effects between predictor variables. We

414 utilize the GridSearchCV function to optimize the parameters for the RF algorithm and set the

415 'neg_log_loss' scoring function as the assessment criterion. The detailed parameter selection and

416 optimization is shown in Supplementary Table S5.

417 Alternative feature encoding approaches. Amino acid sequences provide primary structure

418 information of a protein that work well as feature representations of binary PPIs. Here, we use three

419 commonly used sequence-based encoding schemes including Local Descriptors (LD) (Davies et al.,

420 2008; Yang et al., 2010), Conjoint Triads (CT) (Sun et al., 2017), and Autocorrelation (AC) (Guo et

421 al., 2008; You et al., 2013). Generally, these features cover specific, yet different aspects of protein

422 sequences such as physicochemical properties of amino acids, frequency information of local patterns,

423 and positional distribution information of amino acids.

\section{Evaluation criteria}

426 We conduct 5-fold cross-validation to evaluate the performance of predictive models. Under 5-fold 427 cross validation, all the PPI datasets are equally divided into five non-overlapping subsets and each 
428 subset owns once chance to train/test the model which can provide an unbiased evaluation. We

429 aggregate the following metrics [i.e., accuracy, precision, sensitivity, specificity, F1-score, and area

430 under the precision recall curve (AUPRC)] to evaluate the performance of the proposed method. In

431 particular, we define accuracy as $\frac{T P+T N}{T P+T N+F P+F N}$, precision as $\frac{T P}{T P+F P}$, sensitivity/recall as $\frac{T P}{T P+F N}$,

432 specificity as $\frac{T N}{T N+F P}$ and the F1-score as $\frac{T P}{T P+(F P+F N) / 2}$, where TP/FP is the number of true/false

433 positives and TN/FN is the number of true/false negatives.

\section{Enrichment analysis}

436 Enrichment analysis of viral targets in degree and human complexes. As for degree of human

437 proteins, we obtained 365,284 human PPIs from HIPPIE database (Alanis-Lobato et al., 2017) and

438 calculated the degree of each viral target based on the compiled PPI network. Following the

439 enrichment approach in (Devkota and Wuchty, 2020), the enrichment of viral targets was determined

440 as a function of degree $k$. In particular, $N_{\geq k}$ is the number of viral targets with degree $\geq k$.

441 Randomly sampling $N_{\geq k}$ proteins from the whole set of viral targets, we recalculated the

442 corresponding random number of proteins with degree $\geq k$ (i.e., $N_{\geq k}^{r}$ ). Note, that the random

443 sampling was conducted 100 times and an average of $N_{\geq k}^{r}$ was thus obtained. The enrichment of

444 viral targets that own at least degree $k$ was then defined as $E_{\geq k}=\log _{2}\left(N_{\geq k} / N_{\geq k}^{r}\right)$. In general, $E_{\geq k}>$

4450 points to an enrichment of viral targets with degree $\geq k$.

446 Regarding human complexes, we collected 2,916 human complexes from CORUM (Giurgiu et al.,

447 2019) and calculated the number of participated human complexes of each viral target. Analogously, 
448 the enrichment of viral targets as a function of the number of participated human complexes was

449 determined.

450 GO enrichment analysis. GO annotation data of human proteins were downloaded from 451 http://current.geneontology.org/ (The Gene Ontology Consortium, 2019). Using all human proteins 452 mapped to three categories of GO terms [i.e., Cellular Component (CC), Biological Process (BP) and

453 Molecular Function (MF)] as reference sets, enriched GO terms of viral targets were determined by 454 hypergeometric tests, where corresponding $P$-values were Bonferroni corrected.

455 KEGG pathway enrichment analysis. KEGG pathway data were downloaded from 456 https://www.genome.jp/kegg/ (Kanehisa and Goto, 2000). Using all human proteins in KEGG 457 pathways as a reference set, enriched KEGG pathways of viral targets were identified by 458 hypergeometric tests where corresponding $P$-values were Bonferroni corrected.

459 Essential genes, transcription factors, kinases and post-translational modifications (PTM). We 460 collected 7,063 human essential genes from the Online GEne Essentiality database (OGEE) (Chen et 461 al., 2012) and the Database of Essential Genes (DEG) (Luo et al., 2020). We obtained 1,369 human 462 transcription factors from Gene Transcription Regulation Database (GTRD) (Yevshin et al., 2019) 463 and 508 human kinases from Kinome NetworkX database (Cheng et al., 2014). As for PTM 464 information we obtained 11,670 ubiquitinated proteins, 17,351 phosphorylated proteins, 5,420 465 methylated proteins and 6,992 acetylated proteins from PhosphoSitePlus database (Hornbeck et al., 466 2015). As for the enrichment analysis, we employed hypergeometric tests for each PTM type while 467 all 20,251 reviewed human proteins as of the SwissProt database are considered as the reference set. 
470 In order to build the integrated interaction network for topological analysis, we first collected known

471 protein interactions between the human proteins predicted to interact with SARS-CoV-2 from the

472 HIPPIE database (Alanis-Lobato et al., 2017). In the next step, we combined our predicted

473 human-SARS-CoV-2 PPIs with the known human PPIs into the final interaction network. Moreover,

474 we clustered human proteins within the network based solely on their topological connectivity. We

475 applied the MCODE plugin (Bader and Hogue, 2003) to find clusters of densely interconnected

476 human proteins denoting potential functional modules or parts of pathways (include loops: no;

477 degree cutoff: 2; haircut: no; fluff: no; node score: 0.4; kcore: 2; max depth: 100). Visualizations of

478 the modules (i.e., subnetworks) were carried out with Cytoscape (Shannon et al., 2003). Enrichment

479 analysis for each cluster was performed by using hypergeometric tests, where corresponding

$480 \quad P$-values were Bonferroni corrected, and only the five most enriched GO BP terms and KEGG

481 pathways were considered (Adjusted $P$-value $\leq 0.05$ ) in Figure 6. All the enriched GO terms and

482 KEGG pathways are listed in Supplementary Table S4. 


\section{Supplementary data}

485 Supplementary Table S1. Experimentally verified and predicted human-SARS-CoV-2 PPIs.

486 Supplementary Table S2. Enriched GO terms for experimental and predicted SARS-CoV-2

487 interacting proteins.

488 Supplementary Table S3 Enriched KEGG pathways for experimental and predicted SARS-CoV-2

489 interacting proteins.

490 Supplementary Table S4 Enriched GO terms for identified topological modules.

491 Supplementary Table S5 Parameter selection and optimization of different frameworks.

\section{Acknowledgements}

494 We are grateful to those scientists/developers who enabled the development of the deep learning

495 method by making their data/databases/software freely accessible to the community.

\section{Competing interests}

498 The authors declare that they have no competing interests.

\section{Availability of data and materials}

501 Source code and datasets are available in https://github.com/XiaodiYangCAU/TransPPI/.

\section{$503 \quad$ Funding}

504 This work was supported by the National Key Research and Development Program of China 


\section{Authors' Contributions}

508 SW, XY and ZZ conceived the study and designed the experiment. XY performed the computational

509 analysis and wrote the manuscript. SW and ZZ supervised the study and revised the manuscript. XL,

510 SY and SW provided data preparation and analysis. All authors have read and approved the final

511 manuscript. 


\section{References}

514 Ahmed I, Witbooi P, Christoffels A. 2018. Prediction of human-Bacillus anthracis

515 protein-protein interactions using multi-layer neural network. Bioinformatics 34:4159-4164.

516 doi:10.1093/bioinformatics/bty504

517 Alanis-Lobato G, Andrade-Navarro MA, Schaefer MH. 2017. HIPPIE v2.0: enhancing

518 meaningfulness and reliability of protein-protein interaction networks. Nucleic Acids Res

519 45:D408-D414. doi:10.1093/nar/gkw985

Alguwaizani S, Park B, Zhou X, Huang DS, Han K. 2018. Predicting interactions between virus and host proteins using repeat patterns and composition of amino acids. J Healthc Eng

2018:1391265. doi:10.1155/2018/1391265 

doi:10.1073/pnas.0702332104 via multi-scale convolutional sparse coding for biomedical applications. IEEE Trans Pattern Anal Mach Intell 40:1182-1194. doi:10.1109/TPAMI.2017.2656884 Multifaceted protein-protein interaction prediction based on Siamese residual RCNN. Bioinformatics 35:i305-i314. doi:10.1093/bioinformatics/btz328 database. Nucleic Acids Res 40:D901-D906. doi:10.1093/nar/gkr986 interactome reveals new clues for rational kinase inhibitor discovery and individualized cancer therapy. Oncotarget 5:3697-3710. doi:10.18632/oncotarget.1984 semi-supervised, multi-instance, and transfer learning in medical image analysis. Med Image Anal 54:280-296. doi:10.1016/j.media.2019.03.009 

hepatitis C virus- host cell protein interactions. Mol Biosyst 9:3199-3209. tool. Bioinformatics 29:1357-1358. doi:10.1093/bioinformatics/btt137 interactions between human and HIV proteins. Infect Genet Evol 11:917-923. doi:10.1016/j.meegid.2011.02.022

Dyer MD, Murali TM, Sobral BW. 2008. The landscape of human proteins interacting with viruses and other pathogens. PLoS Pathog 4:e32. doi:10.1371/journal.ppat.0040032 
Ruepp A. 2019. CORUM: the comprehensive resource of mammalian protein complexes — 2019.

Nucleic Acids Res 47:D559-D563. doi:10.1093/nar/gky973 
Mukherjee S, Jacobson M, Malik HS, Fujimori DG, Ideker T, Craik CS, Floor SN, Fraser JS, map reveals targets for drug repurposing. Nature 583:459-468. doi:10.1038/s41586-020-2286-9 doi:10.1093/nar/gku1121 covariance to predict protein-protein interactions from protein sequences. Nucleic Acids Res 36:3025-3030. doi:10.1093/nar/gkn159 doi:10.1093/bioinformatics/bty573 PhosphoSitePlus, 2014: mutations, PTMs and recalibrations. Nucleic Acids Res 43:D512-D520. doi:10.1093/nar/gku1267 
compound-protein affinity through unified recurrent and convolutional neural networks. Conf Mach Learn 14:1188-1196. overcomplete feature learning. Proc Neural Inf Process Syst 1017-1025. scalable unsupervised learning of hierarchical representations. Proc 26th Int Conf Mach Learn 54:609-616. doi:10.1145/2001269.2001295 interactions using primary sequences. Molecules 23:1923. doi:10.3390/molecules23081923 doi:10.1016/j.medj.2020.07.002 human-herpes simplex virus type 1 protein-protein interactions by integrating multiple methods. Quant Biol 8:312-324. doi:10.21203/rs.2.22765/v1 

ECML-PKDD 3-19.

Min X, Zeng W, Chen N, Chen T. 2017. Chromatin accessibility prediction via convolutional long short-term memory networks with k-mer embedding. Bioinformatics 33:i92-i101. doi:10.1093/bioinformatics/btx234 formation by middle east respiratory syndrome coronavirus 4 a accessory protein facilitates viral translation, leading to efficient virus replication. J Virol 92:e00902-18. doi:10.1128/jvi.00902-18 Viral and cellular proteins containing FGDF motifs bind G3BP to block stress Granule formation. PLoS Pathog 11:e1004659. doi:10.1371/journal.ppat.1004659 deep network trained for image classification. eLife 7:e38242. doi:10.7554/eLife.38242 interferon regulatory factor-activating kinases IKKe and TBK-1. J Virol 83:3069-3077. doi:10.1128/jvi.01875-08 

64:39-48. doi:10.1016/j.neunet.2014.08.005 reveals mechanisms of dengue and Zika virus pathogenesis. Cell 175:1931-1945. doi:10.1016/j.cell.2018.11.028 Neural Networks Learn Syst 26:1019-1034. doi:10.1109/TNNLS.2014.2330900 Schwikowski B, Ideker T. 2003. Cytoscape: a software environment for integrated models of biomolecular interaction networks. Genome Res 13:2498-2504. doi:10.1101/gr.1239303 

587:657-662. doi:10.1038/s41586-020-2601-5 using a deep-learning algorithm. BMC Bioinformatics 18:277. doi:10.1186/s12859-017-1700-2 comprehensive and scalable alternative for improving sequence similarity searches. transcriptomics reveals systemic features of rare disease 8:380-394. doi:10.1016/j.cels.2019.04.003 The Gene Ontology Consortium. 2019. The Gene Ontology Resource: 20 years and still GOing strong. Nucleic Acids Res 47:D330-D338. doi:10.1093/nar/gky1055 
284:1097-1105. doi:10.1074/jbc.M805905200 residues in proteins from amino acid sequences using a random forest model with a hybrid feature.

Bioinformatics 25:30-35. doi:10.1093/bioinformatics/btn583 5:e11796. doi:10.1371/journal.pone.0011796 using local descriptors. Protein Pept Lett 17:1085-1090. doi:10.2174/092986610791760306 human-virus protein-protein interactions. Brief Bioinform published online. doi:10.1093/bib/bbaa425. doi:10.1093/bib/bbaa425 interactions through a sequence embedding-based machine learning method. Comput Struct Biotechnol J 18:153-161. doi:10.1016/j.csbj.2019.12.005 on gene transcription regulation - 2019 update. Nucleic Acids Res 47:D100-D105. doi:10.1093/nar/gky1128 
723 amino acid sequences with ensemble extreme learning machines and principal component

724 analysis. BMC Bioinformatics 14:S10. doi:10.1186/1471-2105-14-S8-S10 


\section{Tables}

730 Table 1. Performance of our deep learning architecture (PSSM+CNN+MLP) using 5-fold cross

731 validation.

\begin{tabular}{lllllll}
\hline Human-virus PPI & Accuracy & Precision & Sensitivity & Specificity & F1-score & \\
dataset & $(\%)$ & $(\%)$ & $(\%)$ & $(\%)$ & $(\%)$ & AUPRC \\
\hline Human-HIV & 98.65 & 95.16 & 89.72 & 99.54 & 92.36 & 0.974 \\
Human-Herpes & 95.26 & 77.11 & 68.10 & 97.98 & 72.33 & 0.768 \\
Human-Papilloma & 95.98 & 82.70 & 70.48 & 98.53 & 76.10 & 0.818 \\
Human-Influenza & 96.10 & 84.22 & 70.30 & 98.68 & 76.63 & 0.834 \\
Human-Hepatitis & 93.43 & 69.27 & 49.77 & 97.79 & 57.92 & 0.636 \\
Human-Dengue & 93.29 & 70.02 & 45.85 & 98.04 & 55.41 & 0.605 \\
Human-Zika & 95.41 & 85.17 & 59.94 & 98.96 & 70.36 & 0.746 \\
Human-SARS-CoV-2 & 90.64 & 45.81 & 16.37 & 98.06 & 24.12 & 0.329 \\
\hline
\end{tabular}

732

733 Table 2. Performance comparison of our deep learning architecture and three sequence encoding

734 scheme-based RF methods using 5-fold cross validation.

\begin{tabular}{lcccc}
\hline & \multicolumn{4}{c}{ AUPRC } \\
\cline { 2 - 5 } Human-viral PPI dataset & Our method & LD+RF & CT+RF & AC+RF \\
\hline Human-HIV & 0.974 & 0.972 & 0.970 & 0.972 \\
Human-Herpes & 0.768 & 0.741 & 0.737 & 0.699 \\
Human-Papilloma & 0.818 & 0.740 & 0.724 & 0.656 \\
Human-Influenza & 0.834 & 0.813 & 0.795 & 0.713 \\
Human-Hepatitis & 0.636 & 0.571 & 0.580 & 0.537 \\
Human-Dengue & 0.605 & 0.526 & 0.505 & 0.456 \\
Human-Zika & 0.746 & 0.720 & 0.718 & 0.698 \\
Human-SARS-CoV-2 & 0.329 & 0.371 & 0.350 & 0.314 \\
\hline
\end{tabular}


735 Table 3. Performance comparison of combinations of different feature encodings (PSSM,

736 word2vec+CT one-hot) and our deep learning algorithm (CNN+MLP).

\begin{tabular}{lcc}
\hline & \multicolumn{2}{c}{ AUPRC } \\
\cline { 2 - 3 } Human-viral PPI dataset & PSSM & word2vec+CT one-hot \\
\hline Human-HIV & 0.974 & 0.968 \\
Human-Herpes & 0.768 & 0.734 \\
Human-Papilloma & 0.818 & 0.778 \\
Human-Influenza & 0.834 & 0.808 \\
Human-Hepatitis & 0.636 & 0.587 \\
Human-Dengue & 0.609 & 0.481 \\
Human-Zika & 0.746 & 0.662 \\
Human-SARS-CoV-2 & 0.329 & 0.205 \\
\hline
\end{tabular}

738 Table 4. Performance comparison of our model (PSSM+CNN+MLP) with existing human-virus PPI

739 prediction methods.

\begin{tabular}{|c|c|c|c|c|c|}
\hline Method & $\begin{array}{c}\text { Accuracy } \\
(\%)\end{array}$ & $\begin{array}{c}\text { Precision } \\
(\%)\end{array}$ & $\begin{array}{c}\text { Sensitivity } \\
(\%)\end{array}$ & $\begin{array}{c}\text { Specificity } \\
(\%)\end{array}$ & $\begin{array}{c}\text { F1-score } \\
(\%)\end{array}$ \\
\hline Our model & 94.12 & 97.23 & 90.82 & 97.41 & 93.92 \\
\hline Our previous doc2vec $+\mathrm{RF}^{\mathrm{a}}$ & 93.23 & 95.99 & 90.33 & 96.17 & 93.07 \\
\hline Alguwzizani et al.'s SVM ${ }^{\mathrm{b}, \mathrm{d}}$ & 86.47 & 86.56 & 86.35 & 86.59 & NA \\
\hline DeNovo ${ }^{\mathrm{c}, \mathrm{d}}$ & 81.90 & NA & 80.71 & 83.06 & NA \\
\hline
\end{tabular}

$740{ }^{a}$ The corresponding values were retrieved from (Yang et al., 2020). ${ }^{b}$ The corresponding values were

741 retrieved from (Alguwaizani et al., 2018). ${ }^{\mathrm{c}}$ The corresponding values were retrieved from (Eid et al.,

742 2016). ${ }^{\mathrm{d}}$ NA means the corresponding parameter is not available. 


\section{Figure legends}

745 Figure 1 Introducing a deep learning architecture to predict interactions between viral and human

746 proteins, we designed an end-to-end deep neural network framework. In particular, we designed a

747 Siamese CNN module as high-dimensional sequence embeddings that are fed to the prediction module.

748 In particular, the Siamese architecture of the CNN module allows us to account for residual

749 relationships between interacting viral and human protein sequences through position specific scoring

750 matrices (PSSM), capturing evolutionary relationships between proteins. Such latent protein profile

751 representations of interacting protein sequence pairs are fed to the Siamese CNN module to generate

752 respective high-dimensional sequence embeddings. Finally, output embeddings of two proteins are

753 combined to form a sequence pair vector as the input to a multilayer perceptron (MLP) with an

754 appropriate loss function to predict the presence/absence of an interaction between a viral and a human

755 protein.

757 Figure 2 a Investigating prediction performance we trained our deep learning model on one

758 human-virus PPI data set (rows) and predicted protein interactions in a different human-virus system

759 (columns). Expectedly, the performance of such cross-viral tests dropped considerably in performance

760 compared to training and testing in the same human-viral system. b In a transfer learning step we

761 trained the parameters of the CNN and MLP layers on a source human-virus PPI dataset (rows) and

762 transferred all parameters of CNN layers to initialize a new model to train on a target human-virus PPI

763 dataset (columns). Notably, a relatively rigid transfer learning methodology where we trained the MLP

764 layers with randomly initialized parameters but left the parameters of the feature encoding CNN 
765 untouched (i.e., 'frozen') strongly outperformed baseline performance in a. c In turn, fine-tuning

766 parameters where we trained the MLP layers and retrained the CNN layers using a given target

767 human-viral domain allowed for another marked increase in performance.

769 Figure 3 a Analyzing the predicted network of human-SARS-CoV-2 protein interactions we

770 observed a power-law in the frequency distribution of the number of human proteins that are targeted

771 by a certain number of viral proteins. b Viral targets were enriched in bins of human host proteins

772 that had an increasing number of interactions. c Viral targets were enriched in sets of proteins that

773 participate in an increasing number of human protein complexes. d Analysis of the significance of

774 the overlap between known (left panel) and predicted (right panel) human interacting proteins of

775 SARS-CoV-2 and known human interacting proteins of other pathogens with a hypergeometric test

776 revealed strong similarities with Dengue, Influenza and HIV. The background gene set for the test

777 consisted of all unique human interacting proteins across all pathogens $(\mathrm{N}=6,218$ and $\mathrm{N}=6,200$

778 proteins for comparing with known and predicted human interacting proteins of SARS-CoV-2,

779 respectively).

781 Figure 4 a Venn diagram indicates that overlaps of essential genes and known/predicted viral were

782 statistically significant (*** $P$-value $\leq 0.001$, hypergeometric test). b, $\mathbf{c}$ In turn, overlaps of

783 transcription factors/kinases and predicted/known viral targets were not significant (ns $P$-value $>$

784 0.05). d-f Considering overlaps with post-translational modifications known/predicted viral targets 
785 were significantly enriched with ubiquitinated acetylated, and methylated proteins while

786 phosphorylated proteins were not enriched (*** $P$-value $\leq 0.001$, ns $P$-value $>0.05)$.

788 Figure 5 a The Venn diagram indicates a sizeable overlap of experimentally observed and predicted

789 interactions between proteins of the human host and SARS-CoV-2. b In a quantitative functional

790 analysis of targeted human host proteins, we considered the enrichment of GO terms and KEGG

791 pathways through a hypergeometric test (Bonferroni corrected $P$-value $\leq 0.01$ ). We found that a

792 relatively large shared GO enrichment terms and KEGG enrichment pathways in groups of host

793 proteins that appeared in the experimentally known PPIs and predictions. c In more detail, we

794 observed that enriched GO BP terms in host proteins that were found in the experimental PPIs and

795 predictions were functionally similar.

797 Figure 6 a Combining predictions that we obtained with the transfer learning approach and known

798 human PPIs we determined connectivity-based modules that were subjected to functional

799 interpretation. b Human-SARS-CoV-2 PPI network with enriched GO BP terms and KEGG

800 pathways for each topological module. c SARS-CoV-2 targets a module that involves the centrosome,

801 cell cycle and interferon pathway. SARS-CoV-2 interacts with interferon pathway and presents a

802 conserved region with multiple viral pathogens. A conserved binding motif that nsp13 of

803 SARS-CoV-2 and proteins of various other viral pathogens share suggests that SARS-CoV-2 nsp13

804 protein may interfere with the regulation processes of IFN, supporting antiviral innate immune

805 response. d SARS-CoV-2 targets a module that involves stress granule regulation, RNA processing 

available under aCC-BY-NC-ND 4.0 International license.

806 and protein export, and interacts with stress granule proteins and shows potential interaction patterns

807 by a conserved amino-acid motif in the nsp12 of SARS-CoV-2 and nsp3 proteins of other viruses. 
Sequence pair vector

Sequence embedding vectors

Siamese CNN

(Transfer learning module frozen/fine-tuning)

Protein sequence profiles (PSSM feature)

Multiple sequence alignment

Generated fixed length (L) protein sequences

\section{PPI prediction task \\ Fully connected layers \\ (Multi-layer perceptron)}

\begin{tabular}{|c|} 
vectors \\
\hline $\begin{array}{c}\text { Siamese CNN } \\
\text { (Transfer learning module } \\
\text { frozen/fine-tuning) }\end{array}$ \\
\hline $\begin{array}{c}\text { Protein sequence profiles } \\
\text { (PSSM feature) }\end{array}$ \\
\hline Multiple sequence \\
alignment \\
\hline $\begin{array}{c}\text { Generated fixed length (L) } \\
\text { protein sequences }\end{array}$
\end{tabular}

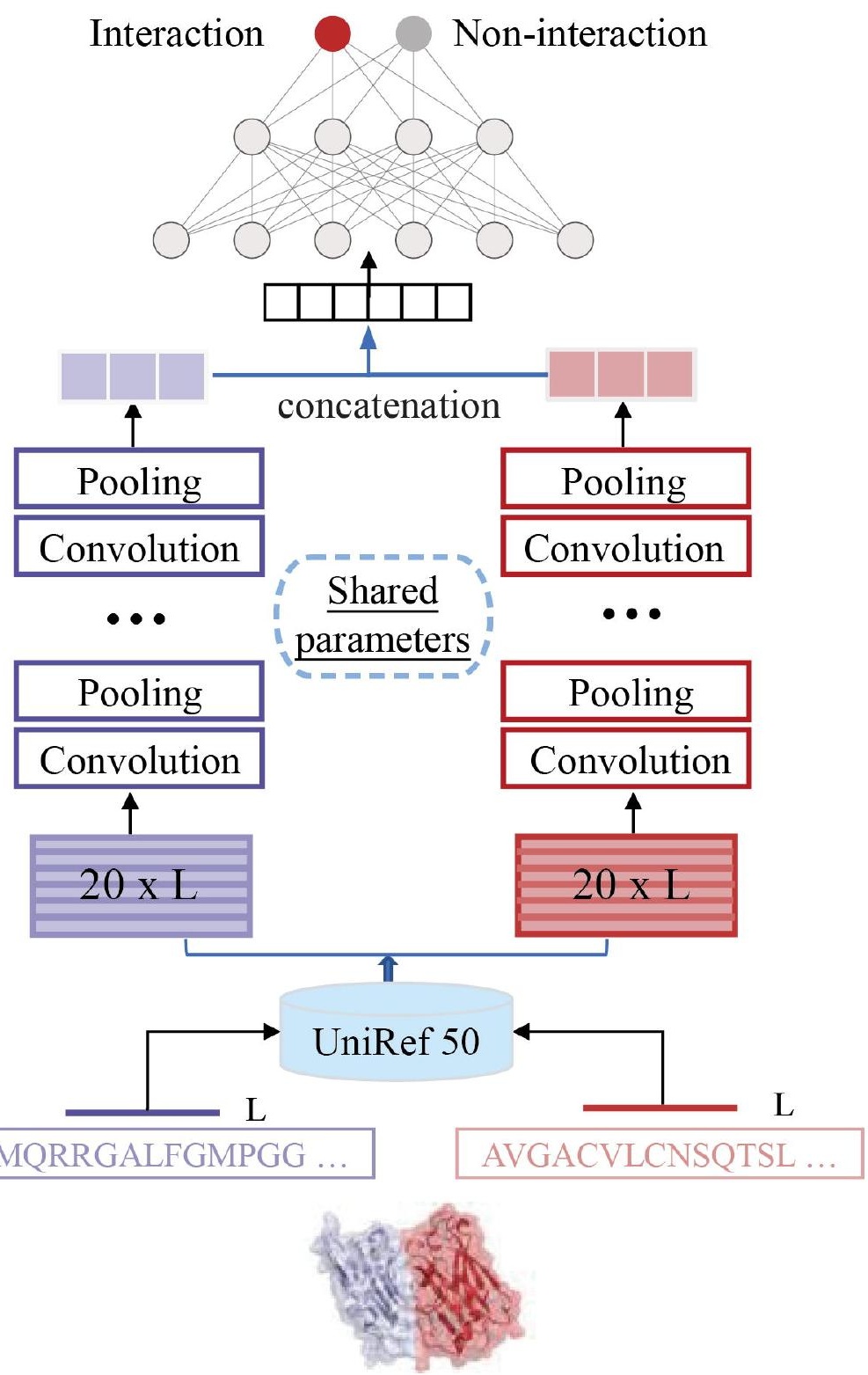




\section{a \\ CROSS-VIRAL TEST}

HIV \begin{tabular}{llll|l|l|l|}
0.209 & 0.182 & 0.227 & 0.121 & 0.166 & 0.149 & 0.181
\end{tabular}

\begin{tabular}{|l|l|l|l|l|l|l|l|l|} 
Herpes & 0.231 & 0.768 & 0.270 & 0.234 & 0.174 & 0.204 & 0.170 & 0.208
\end{tabular}

\begin{tabular}{l|l|l|l|l|l|l|l|l} 
Papilloma & 0.180 & 0.296 & 0.818 & 0.235 & 0.147 & 0.235 & 0.180 & 0.178
\end{tabular}

\begin{tabular}{|l|l|l|l|l|l|l|l|l|} 
Influenza & 0.279 & 0.225 & 0.197 & 0.834 & 0.134 & 0.229 & 0.155 & 0.164 \\
\hline
\end{tabular}

\begin{tabular}{|l|l|l|l|l|l|l|l|l|} 
Hepatitis & 0.171 & 0.164 & 0.156 & 0.174 & 0.636 & 0.176 & 0.105 & 0.131
\end{tabular}

\begin{tabular}{l|l|l|l|l|l|l|l|l} 
Dengue & 0.187 & 0.195 & 0.185 & 0.239 & 0.128 & 0.605 & 0.402 & 0.200
\end{tabular}

\begin{tabular}{l|l|l|l|l|l|l|l|l} 
Zika & 0.133 & 0.122 & 0.140 & 0.151 & 0.084 & 0.284 & 0.746 & 0.152
\end{tabular}

\begin{tabular}{|l|l|l|l|l|l|l|l|l} 
SARS-CoV-2 & 0.240 & 0.164 & 0.145 & 0.171 & 0.097 & 0.186 & 0.178 & 0.329
\end{tabular}

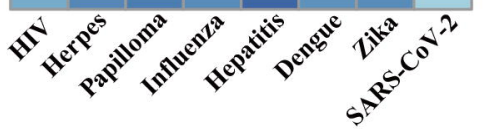

\section{FROZEN}

\begin{tabular}{|l|l|l|l|l|l|l|l|l|}
\hline 0.974 & 0.748 & 0.747 & 0.818 & 0.602 & 0.610 & 0.713 & 0.442 \\
\hline 0.969 & 0.768 & 0.771 & 0.826 & 0.610 & 0.641 & 0.690 & 0.449 \\
\hline 0.970 & 0.765 & 0.818 & 0.824 & 0.587 & 0.619 & 0.716 & 0.430 \\
\hline 0.970 & 0.751 & 0.752 & 0.834 & 0.598 & 0.634 & 0.690 & 0.433 \\
\hline 0.969 & 0.735 & 0.741 & 0.807 & 0.636 & 0.584 & 0.690 & 0.401 \\
\hline 0.970 & 0.728 & 0.745 & 0.810 & 0.593 & 0.605 & 0.695 & 0.417 \\
\hline 0.971 & 0.732 & 0.740 & 0.800 & 0.558 & 0.601 & 0.746 & 0.410 \\
\hline 0.971 & 0.749 & 0.741 & 0.810 & 0.574 & 0.582 & 0.683 & 0.329 \\
\hline
\end{tabular}

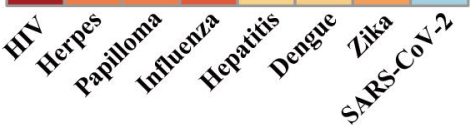

C

\section{FINE-TUNING}

\begin{tabular}{|l|l|l|l|l|l|l|l|}
0.974 & 0.784 & 0.826 & 0.846 & 0.656 & 0.629 & 0.753 & 0.372 \\
\hline
\end{tabular}

\begin{tabular}{|l|l|l|l|l|l|l|l|}
977 & 0.768 & 0.834 & 0.853 & 0.659 & 0.665 & 0.766 & 0.397 \\
\hline
\end{tabular}

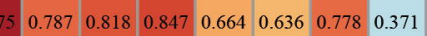

\begin{tabular}{|l|l|l|l|l|l|l|l|}
976 & 0.781 & 0.826 & 0.834 & 0.658 & 0.646 & 0.769 & 0.384
\end{tabular}

\begin{tabular}{|l|l|l|l|l|l|l|l|}
\hline 76 & 0.774 & 0.818 & 0.838 & 0.636 & 0.615 & 0.761 & 0.360 \\
\hline
\end{tabular} \begin{tabular}{|l|l|l|l|l|l|l|l|}
\hline 4 & 0.770 & 0.820 & 0.843 & 0.656 & 0.605 & 0.781 & 0.367 \\
\hline
\end{tabular} \begin{tabular}{|l|l|l|l|l|l|l|l|}
\hline 3 & 0.773 & 0.821 & 0.839 & 0.646 & 0.643 & 0.746 & 0.359 \\
\hline
\end{tabular} \begin{tabular}{|l|l|l|l|l|l|l|l|}
4 & 0.773 & 0.818 & 0.842 & 0.653 & 0.625 & 0.780 & 0.329 \\
\hline
\end{tabular}

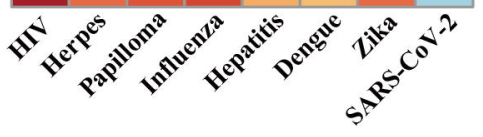




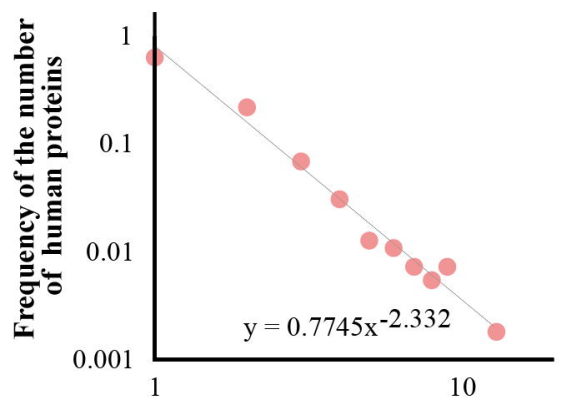

Number of viral proteins b

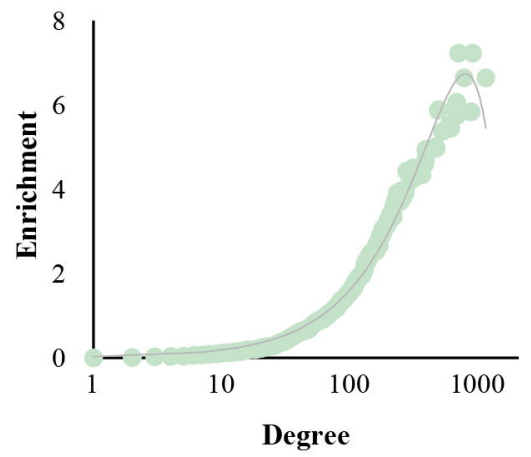

c

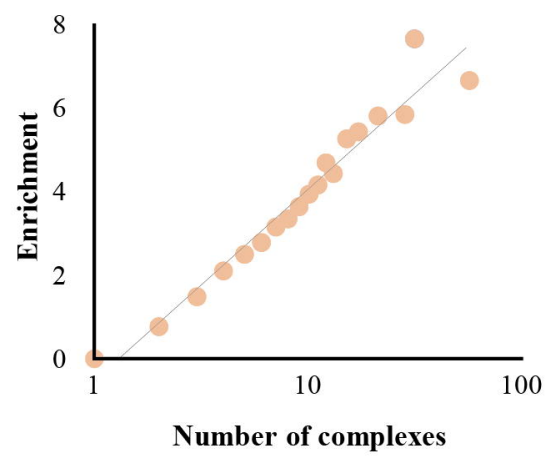

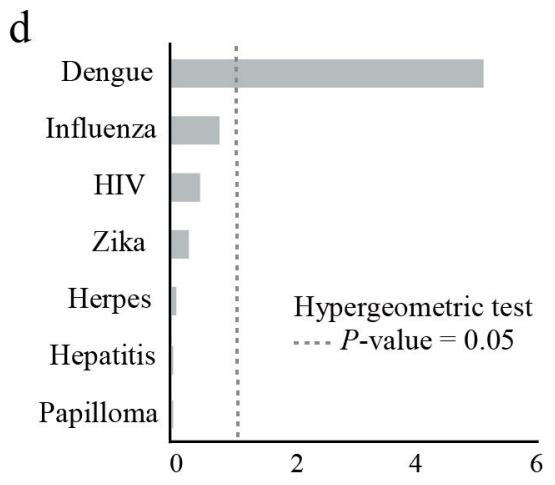

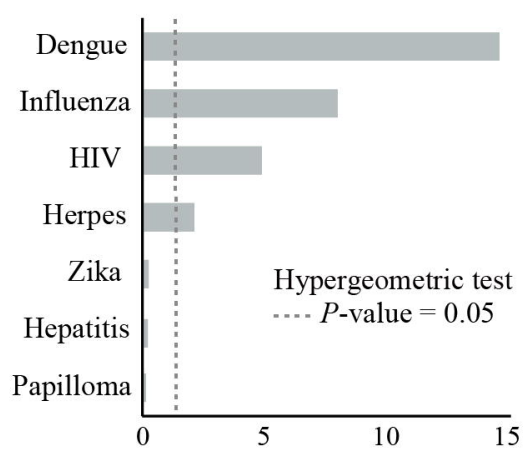

Interactome similarity to known (left panel) or predicted (right panel) human-SARS-CoV-2 interactome $(-\lg (P$-value $))$ 
$a_{\text {prodinted tarcots }} b$

c

Predicted targets Known targets Predicted targets Known targets

Predicted targets Known targets

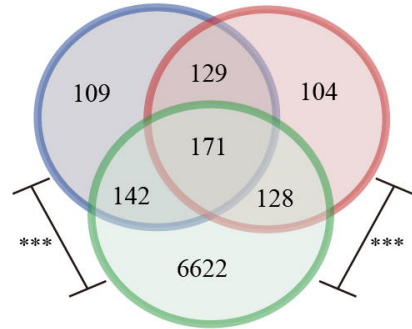

Essential genes

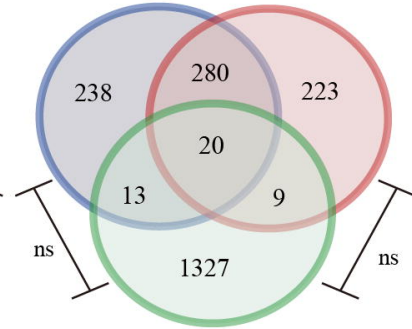

Transcription factors

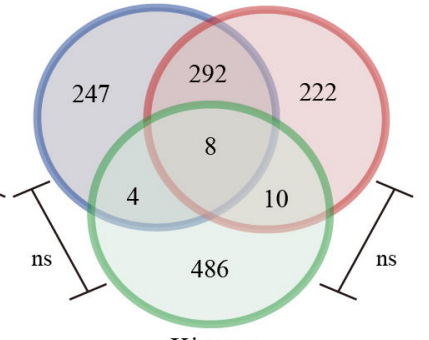

Kinases

d

e $f$

$\mathrm{f}_{\text {Predicted targets Known targets }} \mathrm{g}$

$\mathrm{g}$ Predicted targets Known targets

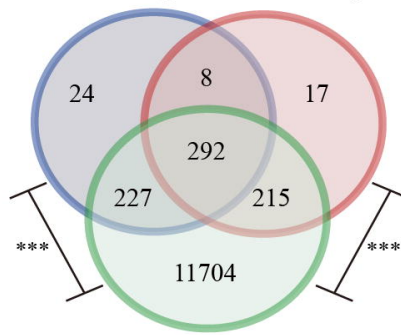

Ubiquitination

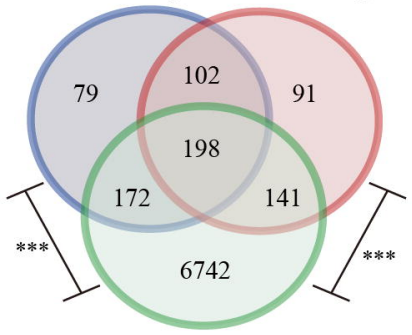

Acetylation

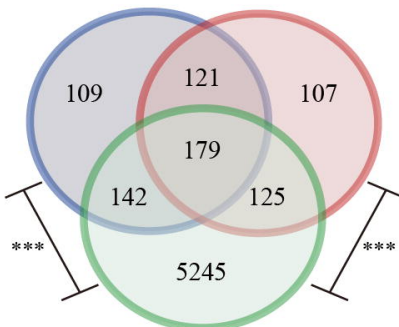

Methylation

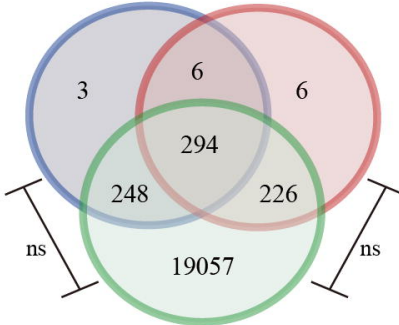

Phosphorylation 


\section{Experimental PPIs}

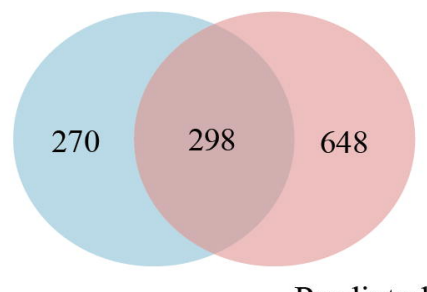

Predicted PPIs b

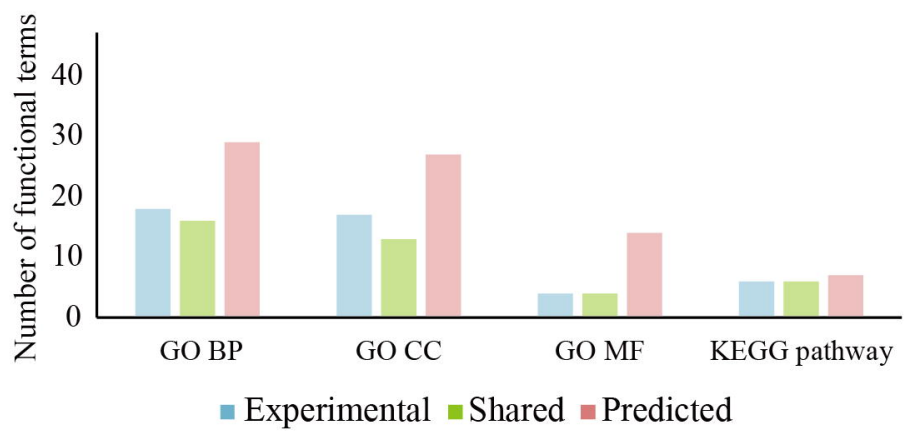

cell redox homeostasis centriole-centriole cohesion chaperone cofactor-dependent protein refolding ciliary basal body-plasma membrane docking cytoplasmic microtubule organization G2/M transition of mitotic cell cycle Golgi to plasma membrane transport intracellular transport of virus mitochondrial calcium ion transmembrane transport mRNA export from nucleus neutrophil degranulation nuclear pore complex assembly nuclear pore organization protein export from nucleus protein folding protein folding in endoplasmic reticulum protein import into nucleus protein $\mathrm{N}$-linked glycosylation via asparagine protein sumoylation protein transport regulation of cellular response to heat regulation of $\mathrm{G} 2 / \mathrm{M}$ transition of mitotic cell cycle regulation of gene silencing by miRNA regulation of glycolytic process regulation of mRNA stability response to unfolded protein stress granule assembly tRNA export from nucleus viral process viral transcription

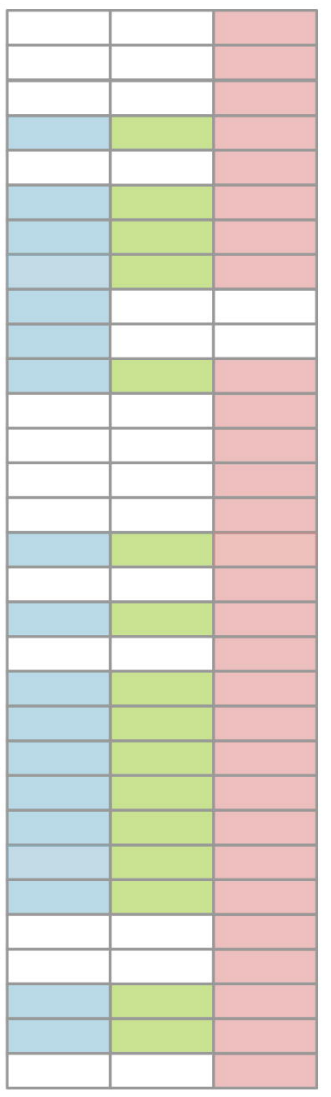

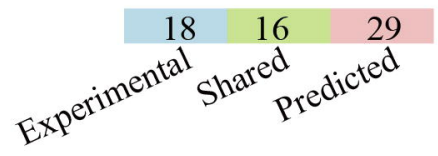


SARS-CoV-2
proteins

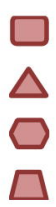

Transfer learning predictions

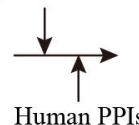

Human-SARS-CoV-2 PPI network
Topological analysis to identify Functional and pathway subnetwork modules enrichments

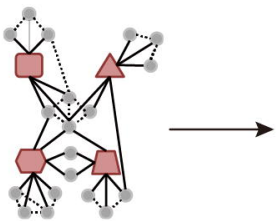

KEGG Pathway

Biological Process b

\begin{tabular}{l} 
Cholesterol metabolism \\
Messenger RNA biogenesis \\
Amyotrophic lateral sclerosis \\
RNA transport \\
protein sumoylation \\
regulation of gene silencing by miRNA \\
regulation of glycolytic process \\
tRNA export from nucleus \\
intracellular transport of virus \\
\hline $\begin{array}{l}5 \quad 10 \quad 15 \quad 20 \quad 25 \\
-\lg (\text { Adjusted } P \text {-value) }\end{array}$
\end{tabular}

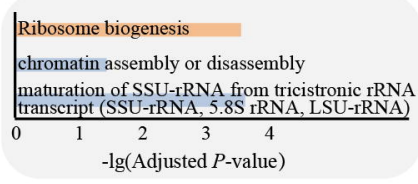

NGDN

$$
\begin{array}{lc}
\multicolumn{2}{l}{\begin{array}{l}
\text { negative regulation of retrograde prote } \\
\text { mRNA stabilization }
\end{array}} \\
\hline 0 & \begin{array}{c}
1 \\
2
\end{array} \\
& -\lg \text { (Adjusted } P \text {-value) }
\end{array}
$$

VDAC2 HNRNPA3 LARP1 AKAP9 HNRNPDL FOXRED2 RAB11B DNAJB4 RPN2 SEL1L

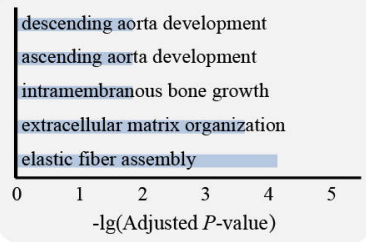

-lg (Adjusted $P$-value)

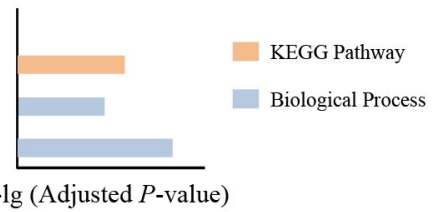

$$
\begin{aligned}
& \begin{array}{ccccccccc}
\text { NGDN } & \text { NOL10 } & \text { FUS } & \text { HTT } & \text { RAB1A RAB8A ATP6V1A } & \text { FBLN5 } & \\
& & \text { RCN1 } & \text { IF3 } & \text { DNAJB1 OS9 } & \text { ERLECl } & & \text { FBN1 } \\
\text { CHD3 } & & \text { TUBGCP2 } & \text { CDK5RAP2 } & \text { PABPC1 } & \text { PCNT } & \text { GRPEL1 } & &
\end{array}
\end{aligned}
$$

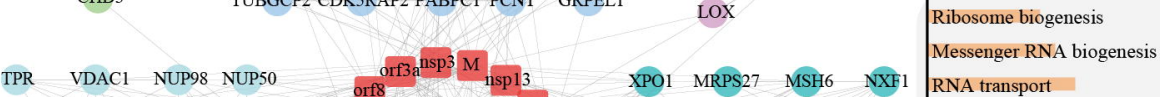

\begin{tabular}{|c|c|c|c|c|}
\hline $\mathrm{n}$ & & Motif: & FGXF & \\
\hline & SARS-CoV-2 nsp12 & 219 & FGDF & 222 \\
\hline Stress granule $:-\mathrm{G}$ & SARS-CoV nsp3 & 1510 & FGYF & 1513 \\
\hline regulation & Bat-CoV nsp3 & 1516 & FGYF & 1519 \\
\hline RNA processing & HCoV-229E nsp3 & 1027 & FGDF & 1030 \\
\hline $\mathrm{N}$ & & 1118 & FGPF & 1121 \\
\hline CSE!L & SFV nsp3 & $451 / 468$ & FGDF & $454 / 471$ \\
\hline Protein export & SINV nsp3 & $490 / 513$ & FGSF & $493 / 516$ \\
\hline M & CHIKV nsp3 & $479 / 497$ & FGDF & $482 / 500$ \\
\hline
\end{tabular}
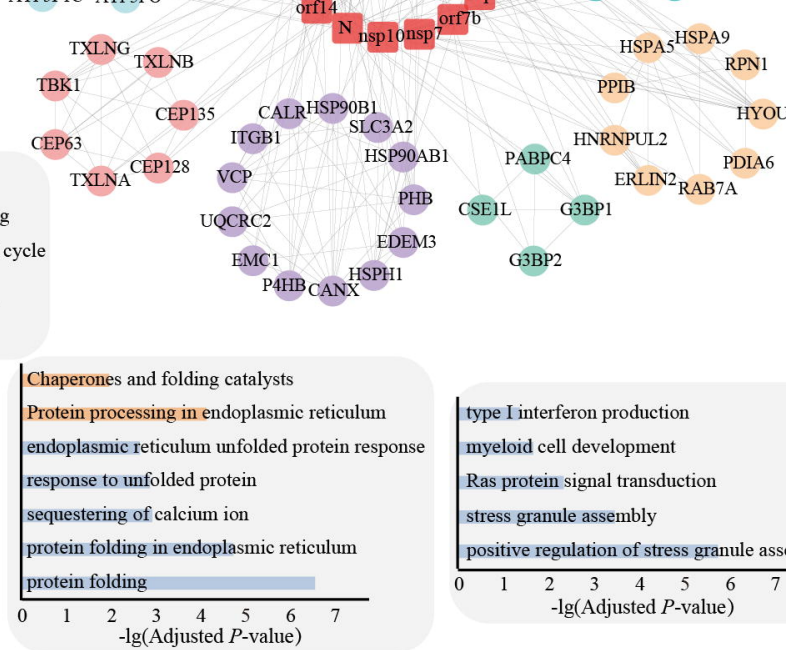

$$
\begin{aligned}
& \text { type I interferon production } \\
& \text { myeloid cell development } \\
& \text { Ras protein signal transduction } \\
& \text { stress granule assembly } \\
& \text { positive regulation of stress granule assembly }
\end{aligned}
$$

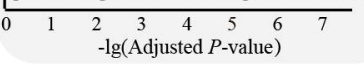

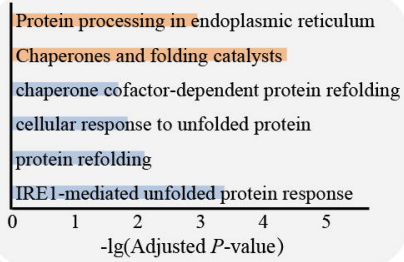

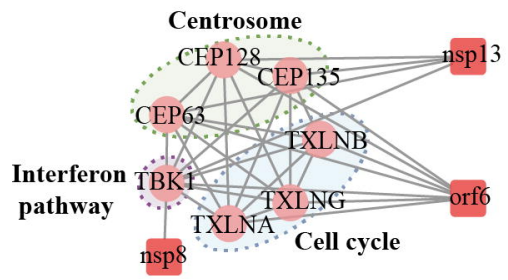

Multiple sequence alignment

$\begin{array}{lrlr}\text { SARS-CoV-2 nsp13 } & 168 & \text { VGKPRPPLNRNYVFTGYRVT } & 187 \\ \text { SARS-CoV nsp13 } & 169 & \text { VGKPRPPLNRNYVFTGYRVT } & 188 \\ \text { BDV P } & 26 & \text { PGSPRPRKVPRNALTQ - - - } & 45 \\ \text { HSV-1 ICP34.5 } & 87 & \text { PPEPAPEARPTAAAPRPRPP } & 106 \\ \text { EBOV VP35 } & 168 & \text { QPPPGPSLYEESAIRGK IES } & 187 \\ & & & \end{array}$

